\title{
Extracting spectral properties from Keldysh Green functions
}

\author{
Andreas Dirks, ${ }^{1,2}$ Martin Eckstein, ${ }^{3}$ Thomas Pruschke, ${ }^{1}$ and Philipp Werner ${ }^{4}$ \\ ${ }^{1}$ Department of Physics, University of Göttingen, D-37077 Göttingen, Germany \\ ${ }^{2}$ Department of Physics, Georgetown University, Washington, DC, 20057 USA \\ ${ }^{3}$ Max Planck Research Department for Structural Dynamics, University of Hamburg-CFEL, Hamburg, Germany \\ ${ }^{4}$ Department of Physics, University of Fribourg, 1700 Fribourg, Switzerland
}

(Received 1 November 2012; published 21 February 2013)

\begin{abstract}
We investigate the possibility to assist the numerically ill-posed calculation of spectral properties of interacting quantum systems in thermal equilibrium by extending the imaginary-time simulation to a finite SchwingerKeldysh contour. The effect of this extension is tested within the standard maximum entropy approach to analytic continuation. We find that the inclusion of real-time data improves the resolution of structures at high energy, while the imaginary-time data are needed to correctly reproduce low-frequency features such as quasiparticle peaks. As a nonequilibrium application, we consider the calculation of time-dependent spectral functions from retarded Green function data on a finite time interval, and compare the maximum entropy approach to direct Fourier transformation and a method based on Padé approximants.
\end{abstract}

DOI: 10.1103/PhysRevE.87.023305

PACS number(s): 02.70.-c, 71.28.+d, 71.10.Fd, 64.60.Ht

\section{INTRODUCTION}

A common strategy to circumvent the oscillatory convergence of integrals in interacting quantum field theories is the Wick rotation to imaginary time. Apart from possible fermionic sign problems, quantum Monte Carlo (QMC) simulations are well conditioned on the imaginary-time axis, and simulations at nonzero temperature using the Matsubara technique are straightforward. However, extracting dynamical properties from imaginary-time data of the single-particle Green function $G(-i \tau)=-i\left\langle T_{\tau} d^{\dagger}(-i \tau) d(0)\right\rangle$ is an ill-posed problem: the singular integral equation

$$
\mathrm{i} G(-\mathrm{i} \tau)=\int d \omega A(\omega) \frac{e^{-\tau \omega}}{1+e^{-\beta \omega}}
$$

has to be solved for the spectral function $A(\omega)$.

If the real-time data for the retarded Green function

$$
G^{\mathrm{ret}}\left(t, t^{\prime}\right)=-i \Theta\left(t-t^{\prime}\right)\left\langle\left\{d(t), d^{\dagger}\left(t^{\prime}\right)\right\}\right\rangle
$$

were known on the entire real-time axis, the spectral function could be obtained from a simple inverse Fourier transform [in equilibrium, $G^{\text {ret }}\left(t, t^{\prime}\right)$ depends only on the time difference $\left.t-t^{\prime}\right]$. The Fourier transform is not ill posed, due to unitarity. Unfortunately, calculating these real-time data for an interacting system is difficult. Monte Carlo techniques can not reach long times, due to a dynamical sign problem which grows exponentially as a function of the maximum real time $t_{\max }$. Nevertheless, as shown in Refs. [1-3], the real-time Green function up to some finite time $t_{\max }$ can be computed accurately using continuous-time Monte Carlo algorithms [4-7] or, in certain parameter regimes, using perturbative weak- [8] or strong-coupling methods [9], as well as Monte Carlo sampling around strong-coupling perturbation theory [10]. This raises the question if the information contained in the real-time correlators for $t, t^{\prime}<t_{\max }$ can be exploited to obtain more reliable spectra using a suitably adapted analytical continuation procedure.

A real need for the "analytical continuation" of realtime Green functions arises in the study of nonequilibrium properties of correlated systems, e.g., lattice systems perturbed by a quench or some field pulse [2,11-13]. In order to characterize the relaxation of these systems, it is often useful to define a time-dependent spectral function

$$
A(\omega, t)=-\frac{1}{\pi} \operatorname{Im} \int_{t}^{\infty} d t^{\prime} e^{i \omega\left(t^{\prime}-t\right)} G^{\mathrm{ret}}\left(t^{\prime}, t\right),
$$

where $G^{\text {ret }}\left(t^{\prime}, t\right)$ now depends on both times individually due to the loss of time-translation invariance. $A(\omega, t)$ is not assured to be positive, but it typically becomes positive a short time after the perturbation. In particular, $A(\omega, t)$ is positive for any quasistationary state. When $A(\omega, t)$ is constant over a time window of width $\Delta t$, then it is positive after averaging over a frequency window of $\Delta \omega \propto 1 / \Delta t$. Under certain assumptions, $A(\omega, t)$ is related to the photoemission and inverse photoemission signal $[14,15]$. Furthermore, in an equilibrium system, Eq. (3) reduces to the familiar spectral function defined in Eq. (1). The challenges in the evaluation of $A(\omega, t)$ are the same as those for equilibrium spectra mentioned above: In practice, simulation results will be limited in time, so direct Fourier transformation will lead to oscillations or a smearing out of spectral features. Therefore, a second question which we want to address is whether maximum entropy or Padé approaches can be used to improve the quality of these time-dependent spectra.

The paper is structured as follows: In Sec. II, we present and test the maximum entropy approach for Keldysh Green functions. In particular, Sec. II B investigates the singular values of the kernel matrix and their dependence on the choice of data points, in Sec. II C we test the analytical continuation procedure for exactly known Green functions, and in Sec. II D we compare the maximum entropy result to spectra obtained by Fourier transformation. A generalization of the Padé analytical continuation procedure to nonequilibrium Green functions is introduced in Sec. III. In Sec. IV, we apply the maximum entropy method to real-time simulation data of equilibrium and nonequilibrium systems, and compare the method to direct Fourier transformation and the Padé procedure. Section V gives a summary and conclusion. 


\section{MAXIMUM ENTROPY APPROACH}

\section{A. Formulation of the problem}

In practice, real-time simulations are carried out on the Keldysh contour $\mathcal{C}[16,17]$ illustrated in Fig. 1 . The Green function is a function of two time indices $t$ and $t^{\prime}$, which may lie on the upper real, the lower real, or the Matsubara branch of the contour. Using the Keldysh-contour time ordering $<_{\mathcal{C}}$, the contour-ordered equilibrium Green function is related to the spectral function through

$$
\begin{aligned}
\mathrm{i} G\left(z, z^{\prime}\right) & =\left\langle\mathrm{T}_{\mathcal{C}} d(z) d^{\dagger}\left(z^{\prime}\right)\right\rangle \\
& =\int d \omega A(\omega) e^{-\mathrm{i}\left(z-z^{\prime}\right) \omega} F_{z, z^{\prime}}(\omega),
\end{aligned}
$$

with the contour-ordered Fermi factor

$$
F_{z, z^{\prime}}(\omega):= \begin{cases}f(-\omega) & \text { if } \quad z^{\prime}<_{\mathcal{C}} z \\ -f(\omega) & \text { else }\end{cases}
$$

and the times $z, z^{\prime}$ located on $\mathcal{C}$. Obviously, Eq. (4) is a generalization of Eq. (1), and extracting $A(\omega)$ from any data set $G\left(z_{i}, z_{j}\right)$ is still an ill-posed problem when the times $z_{i}$ are restricted to the Matsubara branch, or to times smaller than some finite $t_{\max }$.

The essential idea of the maximum entropy (MaxEnt) approach is to infer a most probable spectral function. This is explained in great detail in Ref. [18]. In addition, there exists a vast amount of further literature on the MaxEnt method, focusing both on theoretical issues and on the practical procedures for the analytical continuation of QMC data. An introduction to the MaxEnt method in general can, for example, be found in Ref. [19]. Historically, the approach goes back to the connection between information theory and statistical mechanics as pointed out by Jaynes [20]. The technical aspects of the approach and its applications to the analytic continuation of imaginary-time data have been discussed in a numerous publications, such as Refs. [21-25].

Formally, the problem (4) of analytic continuation can be expressed as a linear equation

$$
D=K A,
$$

where $D$ represents simulation data, $K$ is a linear operator, given by the matrix kernel, applied to the unknown spectral function $A$. The finite set $D$ can be interpreted as a snapshot from a Gaussian random variable with covariance $C$, in the case of a Monte Carlo simulation [18].

An entropy functional $\alpha S[A]$ is subtracted in order to regularize the minimization of $\chi^{2}[D, A]$ with respect to $A$, where

$$
\chi^{2}[D, A]:=\frac{1}{2}(K A-D)^{T} C^{-1}(K A-D) .
$$

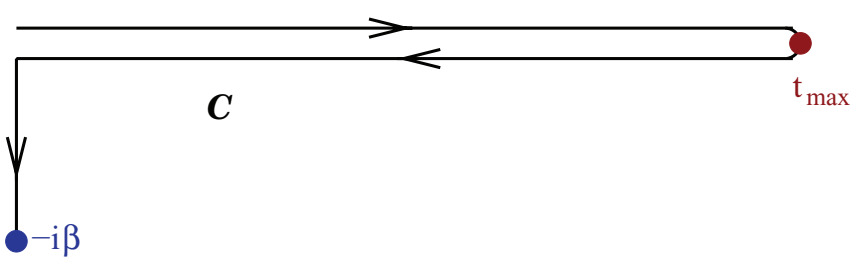

FIG. 1. (Color online) The Schwinger-Keldysh contour $\mathcal{C}$. Arrows indicate the direction of time ordering $<_{\mathcal{C}}$.
The most informative scalar $\alpha$ can be determined by a systematic application of Bayesian inference (classic MaxEnt), or can be averaged over the corresponding Bayesian probability distribution (Bryan's MaxEnt).

The entropy functional is

$$
S[A]=\int d \varepsilon\left[A(\varepsilon)-A_{\mathrm{def}}(\varepsilon)-A(\varepsilon) \ln \frac{A(\varepsilon)}{A_{\mathrm{def}}(\varepsilon)}\right],
$$

where $A_{\mathrm{def}}(\varepsilon)$ is the so-called default model, which contains prior information on the spectrum to be inferred. In this work, we always use a Gaussian default model $A_{\text {def }}(\omega)=$ $\frac{1}{\sigma \sqrt{2 \pi}} \exp \left[-(\omega / \sigma)^{2} / 2\right]$ with width $\sigma=2$. It represents a blurred picture of the true spectral function. $A_{\text {def }}(\omega)$ can be systematically improved, for example, by using appropriately iterated MaxEnt procedures [25], yielding more accurate results. This line of improvements is, however, independent of the structure of the continuation kernel $K$, which is investigated in this work.

The standard numerical algorithm for the MaxEnt inference of spectral functions was developed by Bryan [26]. The kernel is decomposed via a singular value decomposition

$$
K=V \Sigma U^{T},
$$

where $\Sigma=\operatorname{diag}\left(\sigma_{1}, \sigma_{2}, \ldots\right), \sigma_{1} \geqslant \sigma_{2} \geqslant \cdots \geqslant 0$. Through the matrix products, each singular value $\sigma_{i}$ is associated with a direction in $A$ space and a direction in $D$ space. The former is given by one of the "basis functions" for the spectral function and the latter is represented by an entry of $V$.

If $\sigma_{i}$ is large, it provides a channel which transports a comparably large amount of information about $A$. If $\sigma_{i}$ is small, not much information can be gained for the corresponding direction in $A$ space, and the Bayesian approach will not modify a default spectrum with respect to that direction, due to a lack of evidence. A small value of $\sigma$ can only be compensated by small error bars for the corresponding $D$ direction. Hence, the shape of the singular value distribution is an important indicator for the structure of the inverse problem.

\section{B. Singular value distributions}

In this section, we analyze the singular value structure of the integral kernel $K$ in Eq. (6), for various locations of the data set $D$ on the Keldysh contour (the set $A$ is always given by some appropriate discretization of the spectral function). In panel (a) of Fig. 2, the singular value distribution is plotted for the usual inference from imaginary-time data (solid lines), for inverse temperature $\beta=10$. In this case, the data set $D=$ $D_{\text {imag }}$ consists of the values $G\left(-i \tau_{j}, 0\right)$ on $N$ equidistant points on the imaginary-time branch,

$$
D_{\text {imag }}^{N, \beta}=\left\{G\left(-i \tau_{j}, 0\right) \mid \tau_{j}=\beta j / N, j=0, \ldots, N-1\right\} .
$$

The singular values are seen to decay exponentially.

Next, we define a suitable data set for extracting $A(\omega)$ from pure real-time data. We note that the imaginary-time set of input data contains real-time information for both positive (forward direction) and negative (backward direction) frequencies. In the case of real-time data, the situation is different: If only one time ordering of $d, d^{\dagger}$ is taken into account, the MaxEnt method only yields information about the positive or negative frequency range, i.e., the occupied and unoccupied parts of the 

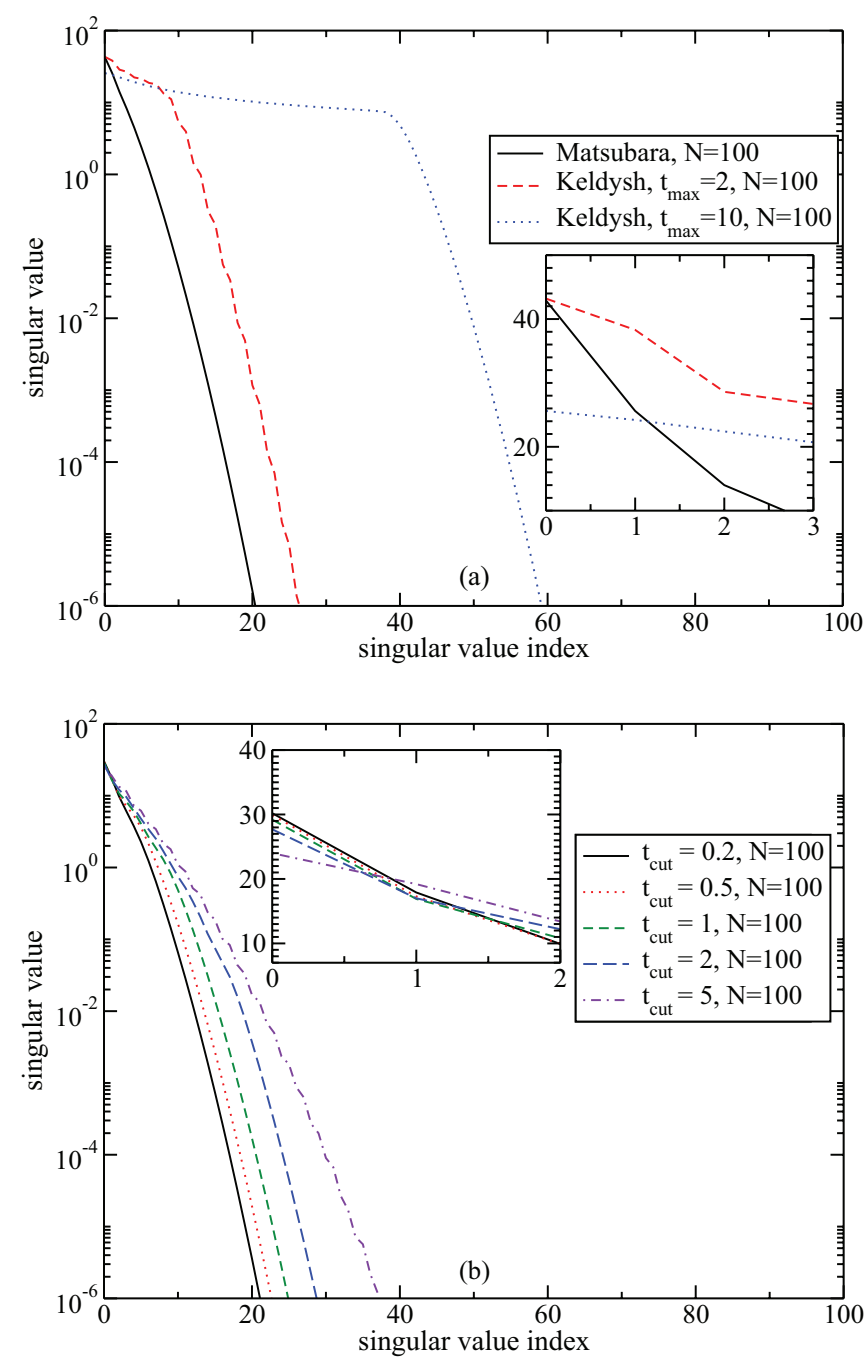

FIG. 2. (Color online) Singular value distributions for $\beta=10$ and indicated number of time points $N$. (a) Matsubara and real-time Green functions. (b) Keldysh-off-diagonal Green functions.

spectrum. It is thus important to consider "lesser" and "greater" (or retarded) Green's functions. We choose $\left(z, z^{\prime}\right)$ values that correspond to both electrons propagating forward in time starting from $t^{\prime}=0, G^{>}(t, 0)=-i\left\langle d(t) d^{\dagger}(0)\right\rangle$, and backward in time starting from $t^{\prime}=t_{\max }$ along the upper real-time branch $G^{<}\left(t, t_{\max }\right)=i\left\langle d^{\dagger}\left(t_{\max }\right) d(t)\right\rangle$, using an equidistant grid of annihilation times:

$$
\begin{aligned}
& D_{\text {real }}^{N, t_{\max }}=\left\{\operatorname{Re} G^{>}\left(t_{j}, 0\right), \operatorname{Im} G^{>}\left(t_{j}, 0\right), \operatorname{Re} G^{<}\left(t_{j}, t_{\max }\right),\right. \\
&\left.\operatorname{Im} G^{<}\left(t_{j}, t_{\max }\right) \mid t_{j}=j t_{\max } 4 / N, j=0, \ldots, N / 4-1\right\} .
\end{aligned}
$$

Due to translational invariance, the $d^{\dagger}$ operator need not be shifted in time. Since we use both the real and imaginary parts of $G^{>}(t, 0)$ and $G^{<}\left(t, t_{\max }\right)$, each time step contributes two real variables to the inference process, and we always use $N$ for the the total number of real variables below.

In contrast to the case of imaginary-time input, the kernel for pure real-time propagators has a distinguished plateau in its singular value distribution, even for rather small $t_{\max }=2$ (dashed line in Fig. 2). As $t_{\max }$ is increased ( $t_{\max }=10$, dotted

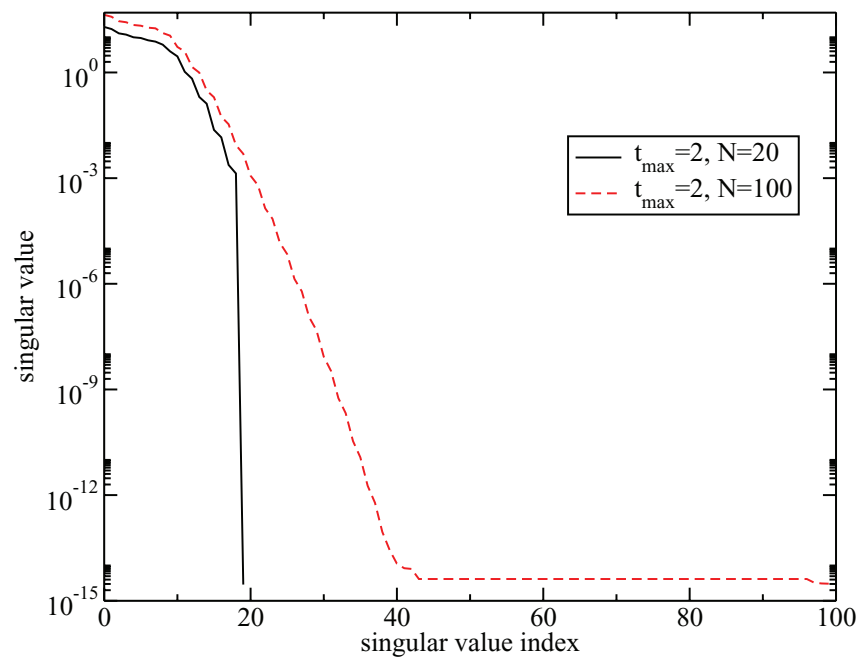

FIG. 3. (Color online) Dependence of real-time singular value distributions on the number of input data $N$ in $D_{\text {real }}^{N, t_{\max }}$.

line), the plateau broadens, and more, equally dominant $A$ directions appear. This is because in the limit $t_{\max } \rightarrow \infty$, the (no longer ill-posed) unitary limit $\sigma_{i} \equiv 1$ is approached.

We next consider singular value distributions for Keldyshoff-diagonal propagators. For symmetry reasons, we can restrict ourselves to mixed Green functions from the upper Keldysh contour to the imaginary branch $G^{\lceil}\left(\tau, t_{\text {cut }}\right)$. We consider fixed values of $t_{\text {cut }} \cdot t_{\text {cut }}=0$ is equivalent to the usual kernel for the Matsubara Green function. As $t_{\text {cut }}$ is increased, the singular value distribution is broadened [Fig. 2(b)], but not as dramatically as in Fig. 2(a). Keeping $\tau=\tau_{\text {cut }}$ fixed yields a structure more similar to the real-time distributions in Fig. 2(a) $\left(\tau_{\text {cut }}=0\right.$ is exactly the same). However, it appears that the plateaux in Fig. 2(a) can not be exceeded.

Figure 3 shows the dependence of the real-time singular value distribution on the number $N$ of time points, for a Keldysh contour of given length $t_{\max }=2$. We find that at low singular value indices, apart from a finite offset, the same shape is obtained. In particular, the same number of singular values is associated with the plateau. Furthermore, the initial descent from the plateau is also identical. However, the singular value distribution for $N=100$ continues to decrease smoothly, whereas the corresponding curve for $N=20$ abruptly jumps to the lowest singular value which is of the order of $10^{-15}$. A similar behavior is also found for other correlators within the Keldysh contour.

We conclude that increasing $N$ does not extend the width of the plateau (once the latter has been established), but merely adds further singular values to the rapidly decreasing tail. Since this decrease is exponential, an increase in $N$ is only useful if the added singular values are larger than the order of magnitude $\varepsilon$ of the numerical error of the data. This dependence is indeed observed in the data analysis presented in the following sections.

\section{Tests of equilibrium spectral functions}

We will first analyze the behavior of the continuation procedure, using artificial data sets that correspond to given spectral functions with sharp peaks or band edges. For this 
purpose, uncorrelated data sets taken from the exact contour Green function are studied, and we assume a uniform error distribution $\varepsilon$, i.e., the covariance matrix is taken to be of the form $C=\operatorname{diag}\left(\varepsilon^{2}\right)$. The performance of the MaxEnt procedures on more realistic data sets will be investigated in Sec. IV.

\section{Rectangular spectrum}

As a first test case, we consider the rectangular spectral function

$$
A_{\text {rect }}(\omega):=\frac{1}{4} \begin{cases}1 & \text { if }|\omega|<2 \\ 0 & \text { else. }\end{cases}
$$

It can be expected that the sharp band edges will be difficult to infer from any finite data set. It is well known that even the inverse Fourier transform, i.e., the unitary limit $t_{\max } \rightarrow$ $\infty$ converges only slowly, and that the convergence is not pointwise. Hence, we consider this an interesting test case for the MaxEnt method. The analytic continuation procedure is tested for inverse temperature $\beta=10$ and for real-branch lengths $t_{\max }=2$ and 10 . The fake variance of all $\mathcal{C}$-contour correlator estimators is set to $\varepsilon^{2}=6 \times 10^{-14}$.

Figure 4 compares the exact spectral function to the $A(\omega)$ obtained from the analytical continuation for different data sets (10) and (11). The total number of real variables is kept constant at $N=100$, i.e., we use either 100 equidistant time steps on the imaginary axis, or 25 time steps in the real-time set $D_{\text {real }}^{N, t_{\max }}$. [We found that the restriction of real input data to the real or imaginary parts of $G\left(t, t^{\prime}\right)$ does not contain sufficient information for an analytical continuation.]

In Fig. 4, it can be seen that using a broad Gaussian default model, the width of which has no significant influence on the results, the MaxEnt solution shows Gibbs ringing artifacts. The frequency of these oscillations is a measure of the accuracy of the inferred spectrum. Surprisingly, already the real-time data from a short Keldysh branch, $t_{\max }=2$, yield more accurate results than the $\beta=10$ Matsubara branch data. As $t_{\max }$ is increased, the approximation of the spectrum becomes even better.

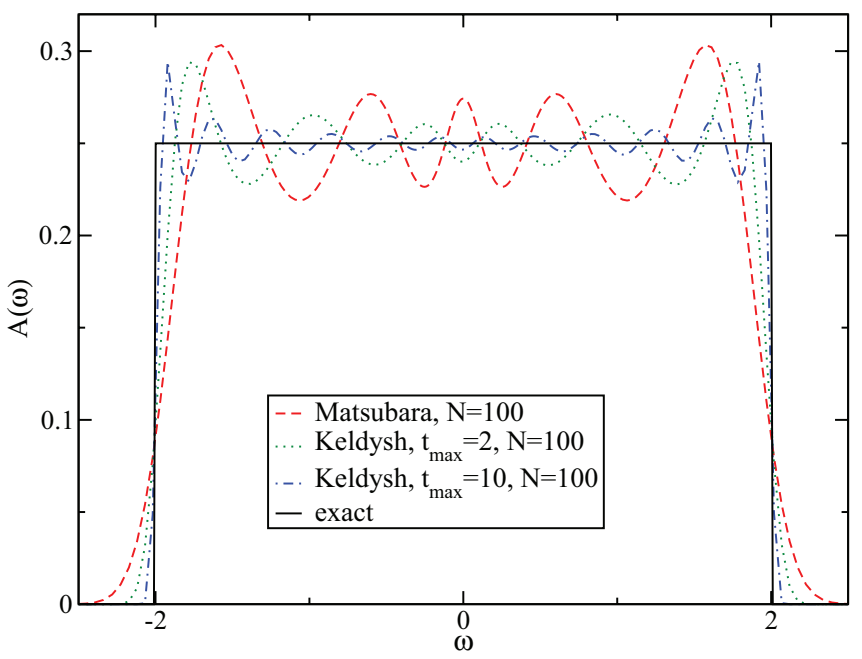

FIG. 4. (Color online) Comparison of rectangular spectra at $\beta=$ 10 for $N=100$ data points located on the Matsubara contour, or on the upper Keldysh contour up to time $t_{\max }=2,10$.
Further increasing the number of data points $N$ does not significantly change the above results. However, lowering the variance $\varepsilon^{2}$ and then raising $N$ systematically yields more accurate spectra for both Matsubara and Keldysh data because in this case the requirements for the identity theorem of complex analysis which guarantees uniqueness are approached systematically. However, the convergence is always exponentially slow, as pointed out in the discussion of the dependence of the singular value distributions on $N$.

\section{Asymmetric triangle}

We next investigate

$$
A_{\text {triang }}(\omega):=\frac{1}{2} \begin{cases}\omega-1 & \text { if }|\omega-2|<1, \\ 0 & \text { else }\end{cases}
$$

as an example for spectra which are not particle-hole symmetric and for which the convergence of the inverse Fourier transform is more rapid than for the rectangular case. The variance is again set to $\varepsilon^{2}=6 \times 10^{-14}$. Results for this scenario are shown in Fig. 5. Judged by the peak position and the steepness at the discontinuity, even the $t_{\max }=0.2$ Keldysh spectrum is already slightly better than the Matsubara spectrum. This indicates that real-time data are good for resolving high-energy features since the triangle is shifted to relatively high energies. As in the case of the rectangular shape, the spectrum improves as $t_{\max }$ is increased.

\section{Multiple peaks}

As a next step towards more realistic situations, we turn to a spectrum with a sharp resonance at $\omega=0$ and two side bands. We model this by superimposing Gaussians

$$
A_{\text {peak }}(\omega):=\sum_{\alpha= \pm 1} c_{\mathrm{sb}} g_{\sigma_{\mathrm{sb}}, \alpha \Omega}(\omega)+c_{\mathrm{res}} g_{\sigma_{\mathrm{res}}, 0}(\omega),
$$

with $c_{\mathrm{res}}=0.1, c_{\mathrm{sb}}=0.45, \Omega=2.0, \sigma_{\mathrm{sb}}=0.5, \sigma_{\mathrm{res}}=0.05$; $g_{\sigma, X}(x):=\frac{1}{\sqrt{2 \pi \sigma^{2}}} \exp \left(-\frac{(x-X)^{2}}{2 \sigma^{2}}\right)$.

As one can see in Fig. 6, real-time data on relatively short contours $\left(t_{\max }=2,10\right)$ are not particularly useful, when sharp low-frequency features such as the given "quasiparticle

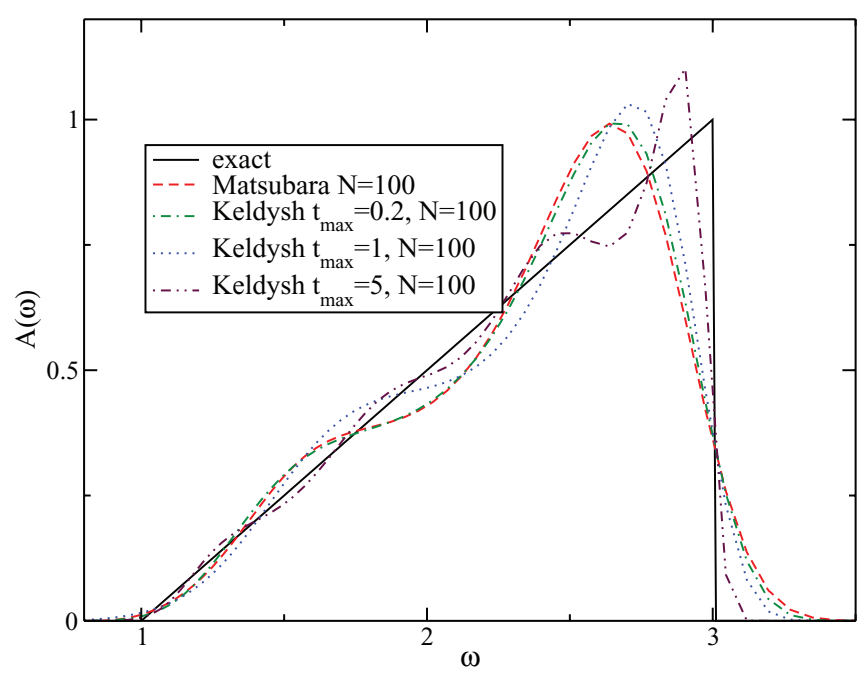

FIG. 5. (Color online) Same as Fig. 4 for the triangular spectrum. 


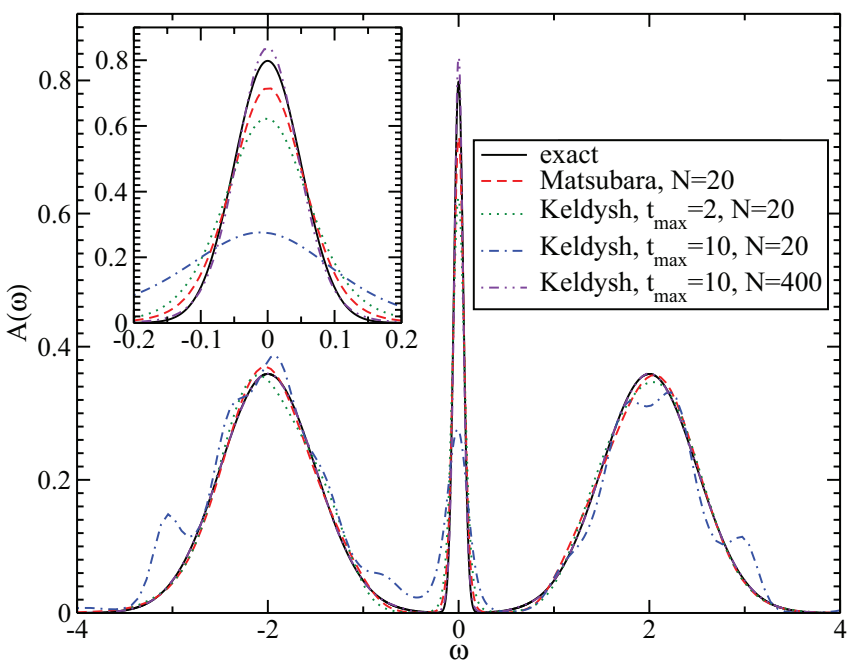

FIG. 6. (Color online) Same as Fig. 4 for the three-peak spectrum.

peak" should be extracted. The Matsubara data and the short- $t_{\max }$ real-time data yield side bands of similar quality, while the imaginary-time data produce a comparable or even better reconstruction of the sharp resonance. Increasing $t_{\max }$ mainly improves the resolution of smooth high-energy features $\left(t_{\max }=10, N=400\right)$. We also observe that using $n=N / 4=$ 5 equidistant time steps on the real contour with $t_{\max }=2$ yields a better spectrum than a similar data set on the $t_{\max }=10$ contour. This finding is compatible with the earlier suggestion that as a function of $N$, a full establishment of the singular value plateau is crucial. However, increasing $N$ at fixed $t_{\max }$ involves only a polynomial increase of the computational effort, so we can always assume that $N$ is sufficiently large for the plateau to be fully developed.

Considering a broader selection of data points from $G^{\lceil}(\tau, t)$, we did not find an improvement of quality of the spectral function compared to pure Keldysh Green functions.

\section{Comparison to direct inversion}

The MaxEnt deconvolution is superior to direct inversion of the Fourier transform for finite Keldysh branches. To demonstrate this, we apply the usual rotation in Keldysh space and consider the retarded Green function defined in Eq. (2). In contrast to Eq. (4), no Fermi factor appears in the transform from the spectral function here:

$$
\mathrm{i} G^{\mathrm{ret}}\left(t-t^{\prime}\right)=\int_{-\infty}^{\infty} d \omega e^{-\mathrm{i} \omega\left(t-t^{\prime}\right)} A(\omega), \quad t>t^{\prime} .
$$

In the limit of a Keldysh contour of infinite length, a Laplace transform restores the spectral function

$$
A(\omega)=-\operatorname{Im} \frac{1}{\pi} \int_{0}^{\infty} d t e^{+\mathrm{i} \omega t} G^{\mathrm{ret}}(t) .
$$

For our contour of finite length $t_{\max }$ a straightforward approximation to the deconvolution problem involves a truncation of the Laplace integral at time $t_{\max }$. Figure 7 shows spectra resulting from this method for the rectangular test spectrum and contour lengths $t_{\max }=2$ and 10 .

Comparison to the finite- $\varepsilon$ MaxEnt results from Keldysh propagators in Fig. 4 shows that the sharp edge is less

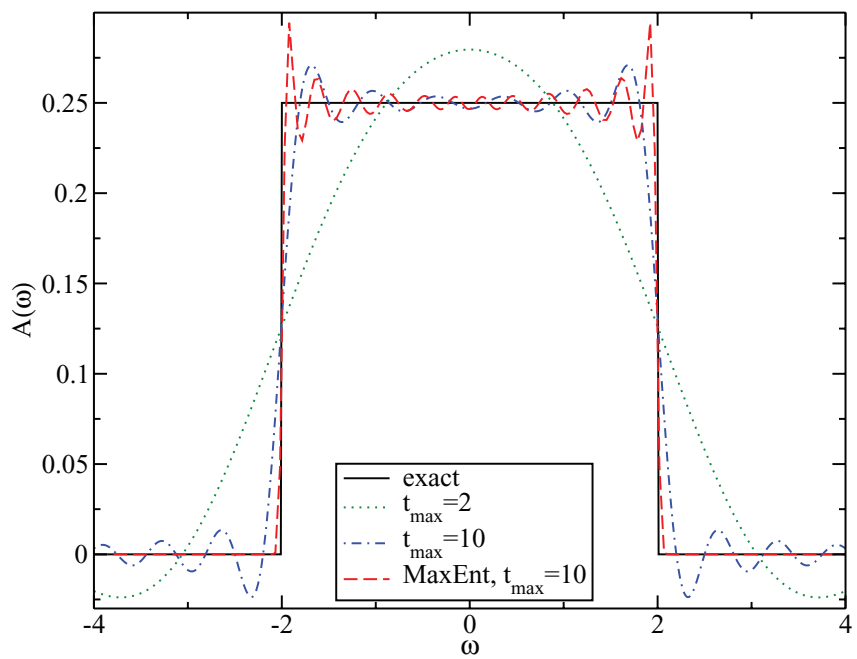

FIG. 7. (Color online) Spectra obtained from the retarded Green function on the Keldysh contour using a truncated Laplace transform $\left(t_{\max }=2\right.$ and 10) and comparison to the exact result. The $t_{\max }=10$ MaxEnt result is included for comparison. See Fig. 4 for a more detailed comparison with MaxEnt data.

well resolved for the direct Fourier transform. Furthermore, unphysical regions of negative spectral weight appear in the case of the truncated Laplace transform, whereas this is avoided by construction in the MaxEnt approach. The amplitude of the ringing oscillations seems to be almost the same as in the MaxEnt case, except near the band edge, where the truncation involves an arbitrary smoothening of sharp structures. Different cutoff procedures for the time integral would yield different smoothenings of the spectrum.

\section{PADÉ APPROXIMATION}

As a second alternative to the MaxEnt approach, we consider the Padé method, which in equilibrium situations is often superior to the MaxEnt method for the analytic continuation of data without, or with very little, stochastic noise. In particular, for the analytical continuation of lowtemperature Matsubara data, it is known to be rather precise at low frequencies [27].

The Padé approximant is constructed as follows: We assume that the values of the Green function are known on a set of $N$ points $z_{n}$ in the complex frequency plane. Conventionally, the $z_{n}$ are given by the Matsubara frequencies $\mathrm{i} \omega_{n}\left[\omega_{n}=(2 n+\right.$ 1) $\pi / \beta]$. The Green function is interpolated with a rational function $C_{N}(z)$ in the form of a continued fraction

$$
C_{N}(z)=\frac{a_{1}}{1+} \frac{a_{2}\left(z-z_{1}\right)}{1+} \cdots \frac{a_{N}\left(z-z_{N-1}\right)}{1},
$$

where the coefficients $a_{i}$ of the continued fraction are computed using a simple recursion formula [28]. A comprehensive introduction to Padé approximants in general can be found in the book by Baker [29].

In this paper, we are in particular interested in the nonequilibrium situation, i.e., the determination of a time-dependent spectral function $A(t, \omega)$ from the real-time retarded Green function on some finite time interval $[t, t+\Delta t]$. For a set of points $z_{n}$ in the complex plane, we first compute an 
approximation $\tilde{G}\left(t, z_{n}\right)$ of the Fourier transformed $G^{\text {ret }}$,

$$
\tilde{G}\left(t, z_{n}\right):=\int_{t}^{t+\Delta t} G^{\mathrm{ret}}\left(t^{\prime}, t\right) e^{\mathrm{i} z_{n}\left(t^{\prime}-t\right)} d t^{\prime} .
$$

This approximation is good if the imaginary part of $z_{n}$ is sufficiently large $\left(\operatorname{Im} z_{n} \gg 1 / \Delta t\right)$. One can then construct a Padé approximant to the function $\tilde{G}(t, z)$ which tends to suppress the artificial oscillatory behavior of spectral functions obtained from direct Fourier transforms (corresponding to $z \rightarrow \omega+\mathrm{iO}^{+}$).

For the approximate Padé method, we consider the following three variants: the points $z_{n}$ are

(1) "imag": fermionic Matsubara frequencies $z_{n}=\mathrm{i} \omega_{n}=$ $\mathrm{i}(2 n+1) \pi / \beta$, with some arbitrarily chosen $\beta$,

(2) "real": real frequencies with some imaginary offset $\mathrm{i} \gamma$, i.e., $z_{n}=n \Delta \omega+\mathrm{i} \gamma$,

(3) "grid": arranged on a $N_{\text {real }} \times N_{\text {imag }}$ grid, where $z_{n_{\text {real }} n_{\text {imag }}}=\omega_{n_{\text {real }}}^{(r)}+\mathrm{i} \omega_{n_{\text {imag }}}^{(i)}$. The lattice is chosen to be equidistant, with $\omega_{n_{\text {real }}}^{(r)}-\omega_{n_{\text {real }}-1}^{(r)}=\Delta \omega$ and $\omega_{n_{\text {imag }}}^{(i)}-\omega_{n_{\text {imag }}-1}^{(i)}=$ $\omega_{1}^{(i)}=: \gamma$.

Figure 8 shows spectra obtained from these different Padé variants, when applied to the rectangular test spectrum. For the approximate Padé versions, we always use the Keldysh contour length $t_{\max }=10$, as well as 100 data points as input for any of the Padé procedures. It is interesting to note that, as compared to the truncated Laplace transform and the MaxEnt approach, the amplitude of the oscillations is significantly smaller for the conventional Padé. However, the resolution of the jump is clearly limited. As $\beta$ is decreased for the Matsubara frequencies, a slight increase of the slope can be observed.

Let us now discuss the approximate Padé method for a time interval length $t_{\max }=10$. By construction, it is closely related to the truncated Laplace transform, whose results are shown in Fig. 7. Nevertheless, features of the original Padé approach are also inherited, depending on how the $z_{n}$ are chosen. In fact, the "imag" solution is much closer to the conventional Padé
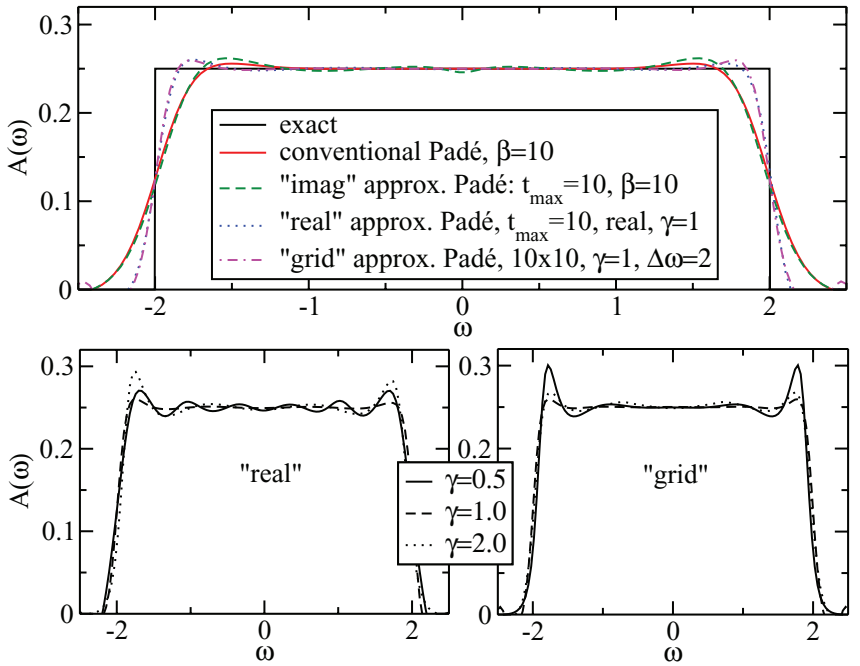

FIG. 8. (Color online) Approximation of the rectangular spectrum with the Padé approach, either conventional or for $t_{\max }=10$. See Figs. 4 and 7 for comparison with MaxEnt and the truncated Laplace transform. solution than to the respective truncated Laplace transform. The undesirable oscillations have been smoothened at the price of a reduced resolution at higher frequencies, while some of the oscillations from the truncated Laplace transform survive. For $\gamma=1$, the "real" and "grid" solutions are almost identical. These results are closer to the exact solution than the truncated Laplace transform, even near the jump, and also exhibit smaller amplitude oscillations. Unfortunately, as shown in the lower panels, the rather unsystematic behavior as a function of $\gamma$ makes it difficult to determine the optimal value of $\gamma$ a priori. We also note that in the "real" variant, the truncated Laplace solution is recovered for $\gamma=0.5$ since in this case all the $z_{n}$ are close to the real axis.

\section{APPLICATION TO DYNAMICAL MEAN FIELD RESULTS}

\section{A. Equilibrium spectra}

As a realistic application, we analyze data obtained for the Kondo lattice model (bandwidth $4, \beta=50, J=1.5$ or 3.0) within dynamical mean field theory in Ref. [30], using the noncrossing approximation (NCA) as impurity solver. We do not want to discuss here the physics of the Kondo lattice model, and to what extent certain high energy features in the spectral function may be an artifact of the NCA. We merely use the results of Ref. [30] as a nontrivial example involving low-energy quasiparticle peaks and high-energy satellites.

In order to apply the MaxEnt method, we again assume a uniform error $\varepsilon$ and set off-diagonal entries of the covariance to zero. This seems justified as long as the systematic numerical error of the simulation data is significantly smaller than $\varepsilon$. In practice, the value of $\varepsilon$ can be rather easily determined by trial and error: if $\varepsilon$ is chosen too small, MaxEnt ceases to converge, whereas if it is chosen too large, the data lack sufficient information. In the following calculations, $\varepsilon$ is chosen slightly larger than the value at which the MaxEnt method breaks down. This moves the systematic numerical errors just into the

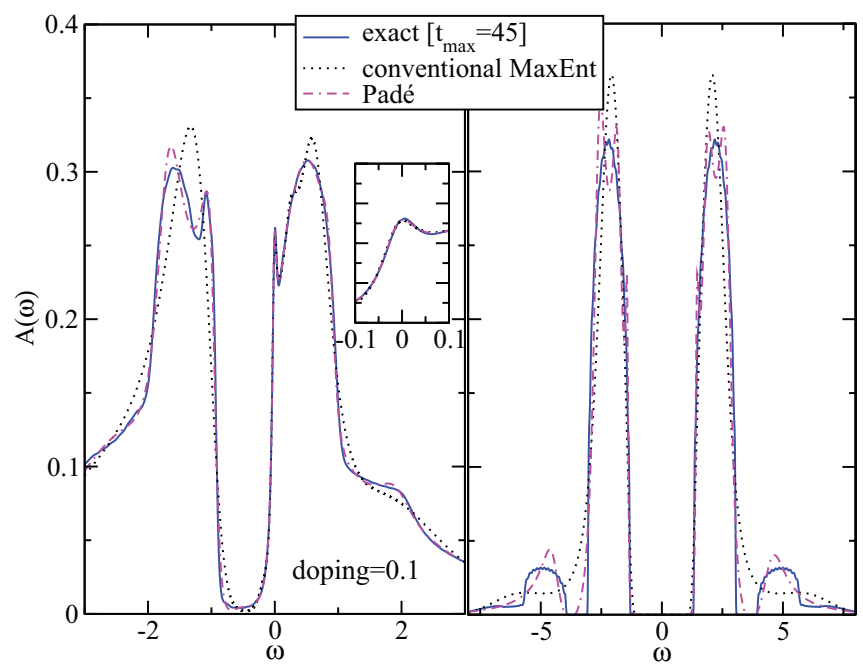

FIG. 9. (Color online) Comparison of Padé and conventional MaxEnt spectra for a Kondo lattice model (bandwidth 4, inverse temperature $\beta=50)$, in the Fermi-liquid regime $(J=1.5$, left $)$ and Kondo insulating regime $(J=3.0$, right $)$. 
$\varepsilon$ range of the Gaussian statistical errors which are formally assumed by the MaxEnt procedure. As input data, we consider the Matsubara Green's functions $G(-i \tau)$ and the retarded (equilibrium) Green functions $G^{\text {ret }}\left(t-t^{\prime}\right)$, as well as data sets containing information from both Matsubara and retarded Green functions.

The NCA data considered here are noise-free low temperature data, and thus ideally suited for the Padé method. In Fig. 9, we compare MaxEnt, Padé, and exact spectra for a half-filled Kondo insulator, and a doped heavy Fermi liquid solution. The "exact" spectra have been obtained from a truncated Laplace transformation with large $t_{\max } \approx 45$. We see that both Padé and Matsubara MaxEnt reproduce the low-energy features and the gap edges very well, but the higher-energy structures can not be accurately resolved.

In Fig. 10, we show in addition to the exact and Matsubara MaxEnt spectra the results from a MaxEnt analytical continuation of real-time data with $t_{\max }=10$ and of a MaxEnt continuation involving both the real-time and the imaginarytime data. The real-time MaxEnt spectra produce the correct
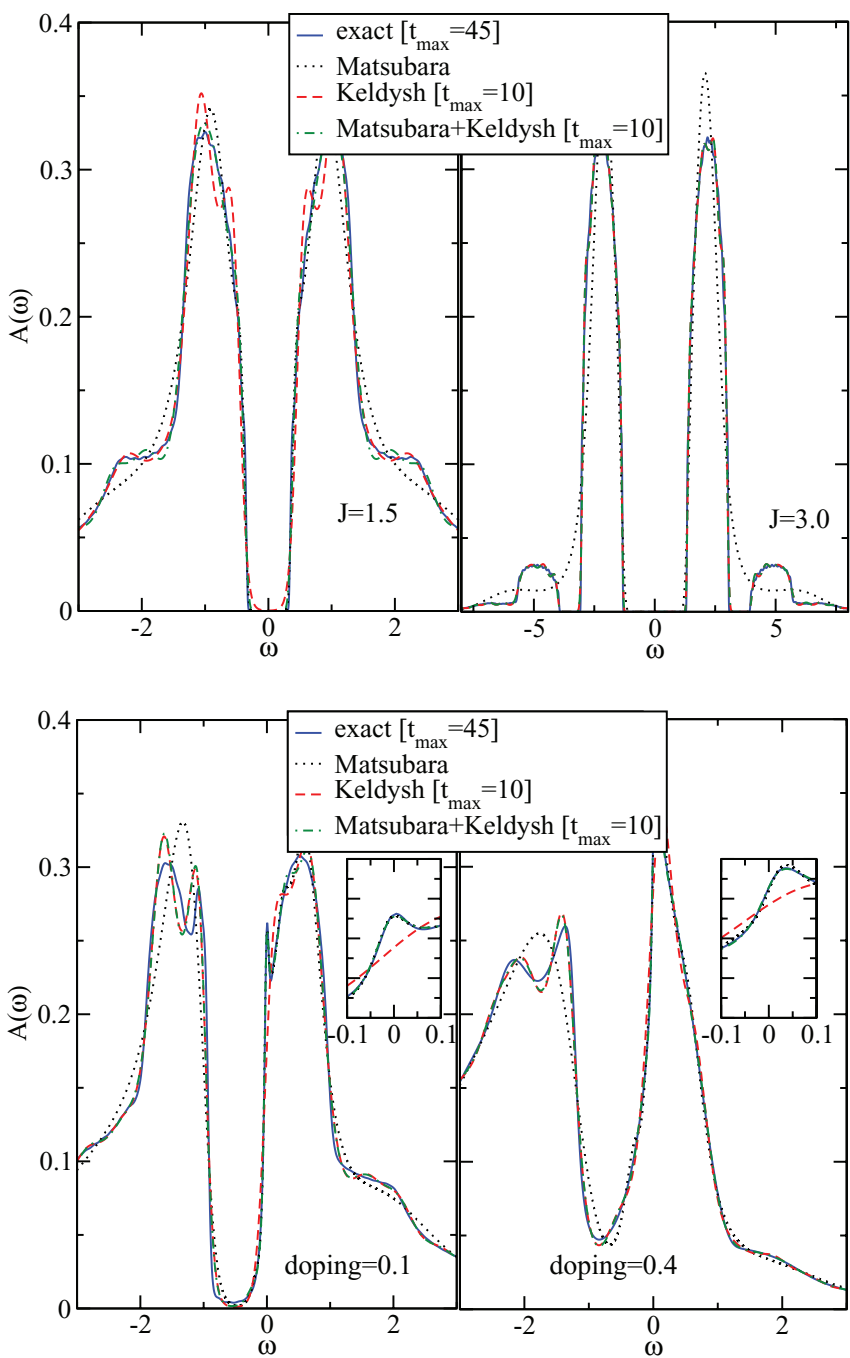

FIG. 10. (Color online) Comparison of MaxEnt solutions for the insulating Kondo lattice model (top, $J=1.5$ and 3.0) and the doped heavy Fermi liquid regime (bottom, $J=1.5$ ). A real-time contour length $t_{\max }=10$ is sufficient to extract the relevant features. high-frequency behavior, and at least qualitatively correct structures in the lower band of the doped system, but they fail to reproduce the quasiparticle peaks. By adding also the imaginary-time data, we can accurately resolve the lowenergy behavior (see insets), in addition to the high-energy structures. The main challenge in the equilibrium case thus remains the calculation of spectral features in the intermediateenergy range, where the convergence to the exact result with increasing $t_{\max }$ is relatively slow.

\section{B. Time-dependent spectra}

To test the ability of the MaxEnt approach to extract timedependent spectra, we again consider results from Ref. [30]. These spectra correspond to a perturbed, doped Kondo lattice model which dissipates energy to a heat bath, and thereby evolves from the high-temperature local moment regime into the low-temperature heavy Fermi liquid regime. Again, we are not interested in the physics here, which has been discussed in Ref. [30], but just in the quality of the spectral functions that can be extracted from the retarded Green functions. In particular, we want to investigate the constraints on $t_{\max }$ for the extraction of a time-dependent spectral function at time $t$ $\left(t<t_{\max }\right)$ since most computational techniques are limited to short time contours.

Figure 11 shows the spectral function $A(\omega, t)$ at a relatively short time $t=6$ after the perturbation. As can be seen, $A(\omega, t)$ is already positive over the whole relevant frequency range. The different curves in the figure correspond to different time windows $[t, t+\Delta t]$ used in the MaxEnt analytical continuation. The result for the largest $\Delta t$ (pink curve) can be considered the exact result. We learn from these results that the high-frequency part converges very quickly with $\Delta t$, while we need at least an interval of length $\Delta t=24$ to get a reasonably accurate result at low energies. (Since this calculation is a real nonequilibrium application, we can not resort to Matsubara data to fix the low-energy part of the spectral function.)

A relevant question is how the MaxEnt approach performs compared to direct Fourier transformation on an identical time interval. While both the MaxEnt method and the direct Fourier
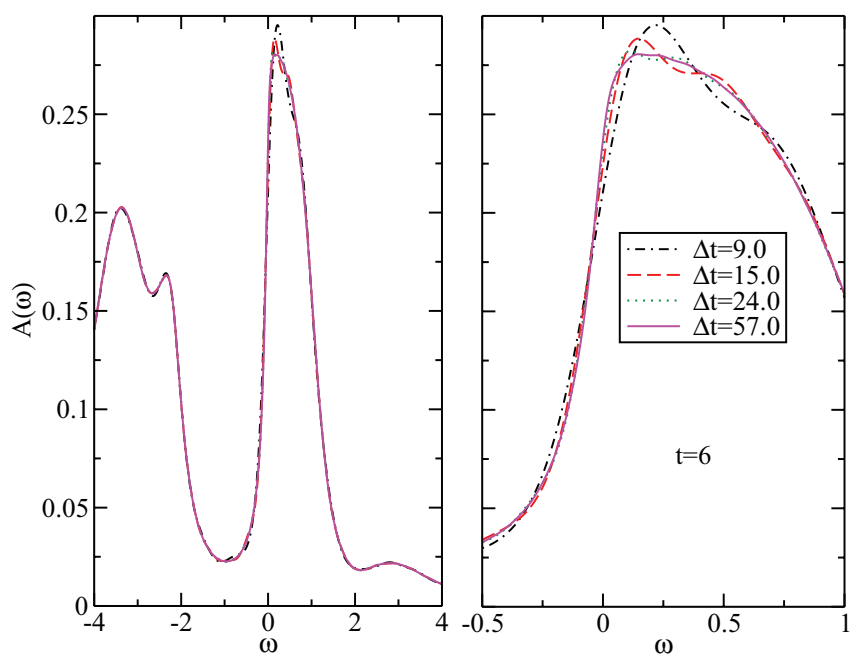

FIG. 11. (Color online) Convergence of $A(\omega, t=6)$ for different time intervals $[t, t+\Delta t]$. 

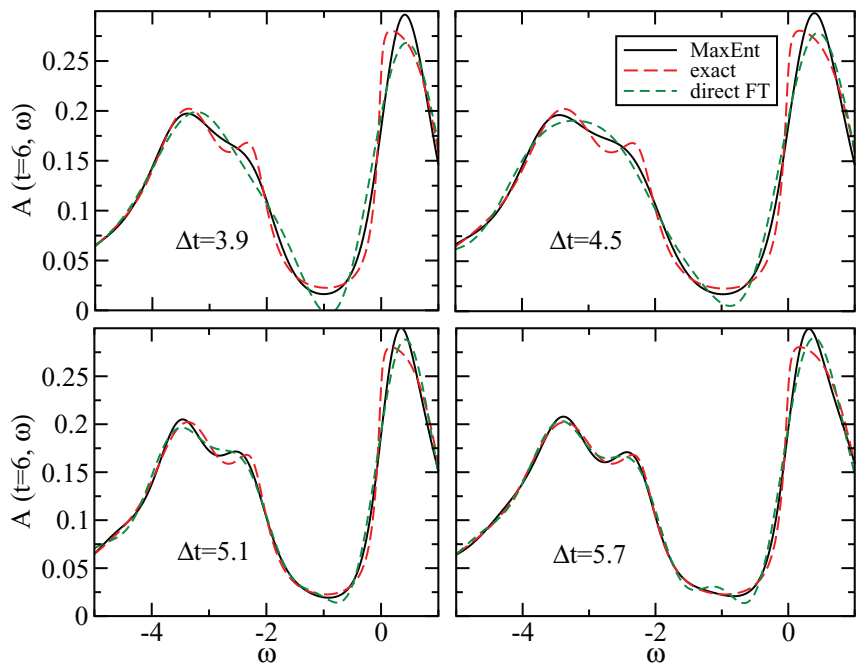
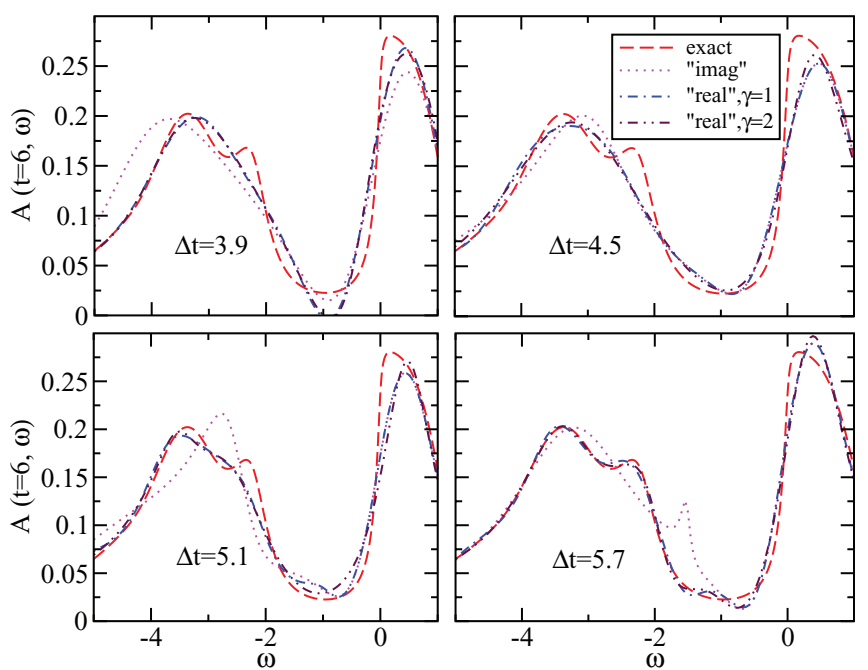

FIG. 12. (Color online) Convergence of nonequilibrium $A(\omega, t=6)$ estimates for different short real-time intervals. In the case of the "imag" modified Padé, we set $\beta=20$ and used 100 data points. For the "real" modified Padé results, we used 100 equidistant data points for $\operatorname{Re} z_{n}$ on the interval $[-10,10]$.

transformation have difficulties reproducing the correct lowenergy peak, the MaxEnt spectra are slightly closer to the correct result.

\section{Convergence of high-energy features}

While the convergence of the MaxEnt result for pure realtime Green function data as a function of $\Delta t$ is slow for the quasiparticle peak, it is interesting to see how the higher-energy features of the nonequilibrium spectral function converge to the exact result.

Figure 12 shows a comparison of the MaxEnt result to the direct Fourier transform and the modified Padé method (discussed in Sec. III) for short time intervals with lengths $\Delta t \in[3.9,5.7]$. For $\Delta t=5.7$, MaxEnt and direct Fourier transformation produce the correct solution, except for in the low-energy region $-0.5 \lesssim \omega \lesssim 0.5$ (left panels), while the modified Padé method for the imaginary-time interval yields spurious results (right panels). For shorter interval lengths, MaxEnt appears to be slightly superior to both direct Fourier transform (FT) and modified Padé. All qualitative features of the spectrum, including the double-peak structure in the lower band, start to become visible in the MaxEnt solution at $\Delta t=4.5$, whereas the emergence of similar structures in the direct FT spectrum at $\Delta t=5.1$ may still be interpreted as part of the overall oscillatory behavior of that solution. One may thus argue that MaxEnt reduces the necessary interval length from $\Delta t=5.7$ to $\Delta t=4.5$ and thus allows us to compute time-dependent spectra up so slightly longer times. Depending on the application, this could save considerable effort since numerical algorithms typically scale badly (at least as the third power) with the length of the contour. The effect of the modified Padé for $z_{n}$ on parallel lines to the real axis can to a large extent be interpreted as a smoothening procedure for the direct FT. In particular, the double-peak structure in the direct FT solution at $\Delta t=5.1$ is strongly suppressed, but still slightly visible. However, the peaks are less pronounced than those of the MaxEnt solution at $\Delta t=4.5$. The behavior of the "grid" variant is very similar to the "real" variant, except that the "grid" choice can not resolve the double-peak structure of the lower band (not shown). When the number of real frequency points is increased, the "grid" result converges to the "real" result. Due to numerical instabilities of the Padé procedure, one is however typically limited to less than 400 grid points.

\section{CONCLUSION}

We analyzed the usefulness of Keldysh real-time Green function data for the computation of equilibrium spectral functions of interacting quantum many-body systems. The ill-posed nature of the inversion that corresponds to the determination of the spectral function is reflected in the distribution of singular values of the respective continuation kernel. The conventional Wick rotation of Matsubara data yields an exponentially decaying singular value distribution. We found that including a finite real-time branch to the imaginary-time contour adds a plateau to this distribution, which broadens as the length of the real-time contour is increased. This plateau significantly alleviates the inversion even for small contour lengths. A further analysis showed that the main gain is a more precise high-frequency information of the spectral function. For short contours, the real-time data, however, provide rather crude results at low frequencies. In order to obtain more accurate estimates of low-energy features such as quasiparticle peaks, it is therefore necessary to include data from the Matsubara branch. With the small frequencies well covered by the Matsubara branch and the high frequencies well covered by a short Keldysh branch, the biggest challenge remains the resolution at intermediate energies.

Our analysis of NCA spectra for the Kondo lattice model suggests that in order to resolve intermediate energies reasonably well, the length of the real-time contour has to be at least $t_{\max }=10$ (in units where the bandwidth is 4 ). In real-time Monte Carlo simulations, it is difficult to reach these times, at least in parameter regimes where low-order perturbation theory is not reliable. It thus appears that the exponential 
increase in the computational cost with increasing $t_{\max }$ in real-time Monte Carlo schemes outweighs the benefits from an alleviated MaxEnt analytical continuation. Nevertheless, the MaxEnt analytical continuation of Keldysh Green functions can be a useful and superior alternative to Padé approximants for semianalytic methods such as higher-order strong-coupling perturbation theory. These methods are very useful in certain parameter regimes, such as elevated temperature, or intermediate to strong interactions. While their implementation on the real-frequency axis is challenging, calculations on the Keldysh contour scale polynomially with $t_{\max }$ and the contour lengths needed for reliable MaxEnt analytical continuation can be reached.

In the calculation of nonequilibrium spectra, it is no longer possible to include the Matsubara branch into the MaxEnt procedure. As a consequence, the resolution of low-energy features of the spectrum worsens dramatically if only short-time data are available. Nevertheless, a comparison to alternative, more straightforward techniques, i.e., direct Laplace transform and the modified Padé approach, showed that MaxEnt yields slightly more reliable solutions for these spectra. The relevant structures could be established for somewhat shorter time intervals than in the case of the extended Padé approaches.

On a conceptual level, the MaxEnt is superior to the direct Laplace transform and the generalized Padé approach. In practical applications, however, because the advantage of a MaxEnt continuation appears to be rather subtle, it will very much depend on the details of the utilized many-body approach whether an application of MaxEnt is worth its effort. In any case, the comparison of different approaches can help in deciding which features of a nonequilibrium spectrum are trustworthy.

\section{ACKNOWLEDGMENTS}

We acknowledge funding from the Deutsche Forschungsgemeinschaft (DFG) via SFB 602, SNF grant PP0022-118866, and FP7/ERC starting Grant No. 278023. This work also profited from the GoeGrid initiative and the Gesellschaft für Wissenschaftliche Datenverarbeitung Göttingen (GWDG).
[1] M. Eckstein, M. Kollar, and P. Werner, Phys. Rev. Lett. 103, 056403 (2009).

[2] M. Eckstein, M. Kollar, and P. Werner, Phys. Rev. B 81, 115131 (2010).

[3] P. Werner, T. Oka, M. Eckstein, and A. J. Millis, Phys. Rev. B 81, 035108 (2010).

[4] A. N. Rubtsov, V. V. Savkin, and A. I. Lichtenstein, Phys. Rev. B 72, 035122 (2005).

[5] P. Werner, A. Comanac, L. de' Medici, M. Troyer, and A. J. Millis, Phys. Rev. Lett. 97, 076405 (2006).

[6] L. Mühlbacher and E. Rabani, Phys. Rev. Lett. 100, 176403 (2008).

[7] P. Werner, T. Oka, and A. J. Millis, Phys. Rev. B 79, 035320 (2009).

[8] N. Tsuji, M. Eckstein, and P. Werner, arXiv:1210.0133.

[9] M. Eckstein and P. Werner, Phys. Rev. B 82, 115115 (2010).

[10] E. Gull, D. R. Reichman, and A. J. Millis, Phys. Rev. B 84, 085134 (2011).

[11] M. Eckstein and P. Werner, Phys. Rev. B 84, 035122 (2011).

[12] N. Tsuji, T. Oka, H. Aoki, and P. Werner, Phys. Rev. B 85, 155124 (2012).

[13] P. Werner, N. Tsuji, and M. Eckstein, Phys. Rev. B 86, 205101 (2012).

[14] M. Eckstein and M. Kollar, Phys. Rev. B 78, 245113 (2008).

[15] J. K. Freericks, H. R. Krishnamurthy, and Th. Pruschke, Phys. Rev. Lett. 102, 136401 (2009).

[16] J. Schwinger, J. Math. Phys. 2, 407 (1961).
[17] For an introduction into the Keldysh formalism, see, e.g., H. Haug and A.-P. Jauho, Quantum Kinetics in Transport and Optics of Semiconductors, 2nd ed. (Springer, Berlin, 2008).

[18] M. Jarrell and J. E. Gubernatis, Phys. Rep. 269, 133 (1996).

[19] N. Wu, The Maximum Entropy Method, Springer Series in Information Sciences (Springer, Berlin, 1997).

[20] E. T. Jaynes, Phys. Rev. 106, 620 (1957).

[21] R. N. Silver, D. S. Sivia, and J. E. Gubernatis, Phys. Rev. B 41, 2380 (1990).

[22] W. von der Linden, R. Preuss, and W. Hanke, J. Phys.: Condens. Matter 8, 3881 (1996).

[23] M. Jarrell, A. Macridin, K. Mikelsons, and D. G. S. P. Doluweera, in Lectures on the Physics of Strongly Correlated Systems XII, AIP Conference Proceedings, Vol. 1014, edited by A. Avella and F. Mancini (AIP, New York, 2008), p. 34.

[24] S. Fuchs, Th. Pruschke, and M. Jarrell, Phys. Rev. E 81, 056701 (2010).

[25] O. Gunnarsson, M. W. Haverkort, and G. Sangiovanni, Phys. Rev. B 81, 155107 (2010)

[26] R. K. Bryan, Eur. Biophys. J. 18, 165 (1990).

[27] J. Otsuki, H. Kusunose, P. Werner, and Y. Kuramoto, J. Phys. Soc. Jpn. 76, 114707 (2007).

[28] H. J. Vidberg and J. W. Serene, J. Low Temp. Phys. 29, 179 (1977).

[29] George A. Baker, Padé Approximants, 2nd ed. (Cambridge University Press, Cambridge, UK, 2010).

[30] P. Werner and M. Eckstein, Phys. Rev. B 86, 045119 (2012). 